\title{
Commentary: Radial or tangential
}

\author{
Dawn S. Hui, MD, ${ }^{a}$ and Richard Lee, MD, MBA ${ }^{b}$
}

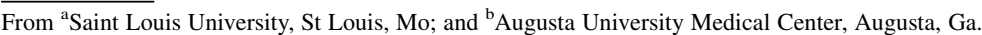 \\ Disclosures: Authors have nothing to disclose with regard to commercial support. \\ Received for publication Nov 7, 2018; accepted for publication Nov 7, 2018; available ahead of print Dec 12, \\ 2018. \\ Address for reprints: Dawn S. Hui, MD, 3635 Vista Ave, DT 13th, Floor, St Louis, MO 63110 (E-mail: dawn.hui@ \\ health.slu.edu) \\ J Thorac Cardiovasc Surg 2019;158:451-2 \\ 0022-5223/ $\$ 36.00$ \\ Copyright (C 2018 by The American Association for Thoracic Surgery \\ https://doi.org/10.1016/j.jtcvs.2018.11.011
}

Studies of coronary revascularization may report a number of end points: angiographic graft patency, need for repeat revascularization, cardiovascular events, cardiovascular or all-cause mortality, event-free survival, and composite outcomes. There are some finer subtleties to some of these end points. For example, angiographic patency may refer to complete graft occlusion or to functional graft occlusion, which primarily affects the radial artery (RA) (7\% vs $0.9 \%$ of saphenous vein grafts). ${ }^{1}$ The need for repeat revascularization may be due to nonpatent grafts or to new native vessel lesions. Study design in multiple arterial revascularization (MAR) should also consider patient selection and operative decisions such as skeletonization of internal thoracic arteries, in situ configuration, native vessel stenosis percentage, and territory grafted. For MAR, completed randomized controlled trials (RCTs) have not yet provided clear answers to these questions. Past RCTs have been underpowered because of failure to recruit or insufficient follow-up. ${ }^{1,2}$ Another concern is generalizability; the Radial Artery Patency and Clinical Outcomes trial ultimately enrolled only $24.9 \%$ of those screened for randomization to RA versus saphenous vein graft (SVG). ${ }^{3}$

In this edition of the Journal, Ruttman et $\mathrm{al}^{4}$ report on their single-center experience with MAR using the left internal thoracic artery (LITA) or bilateral internal thoracic arteries (BITA), with some proportion of patients also having an RA graft. On the basis of their findings, the authors assert that their study provide "clear answers to 2 important issues" in MAR, namely, use of the right internal thoracic artery versus RA as the second arterial graft and patency of RA versus SVG. The statistical analysis was designed to navigate many of the issues described. However, we caution the readers about the strength of evidence to support the authors' conclusions and other points raised in the discussion.

The total cohort had 1654 patients, of whom 55\% received BITA and $45 \%$ received LITA. Preoperative characteristics differed between the groups, some favoring the BITA group (less diabetes, lower creatinine, less severe

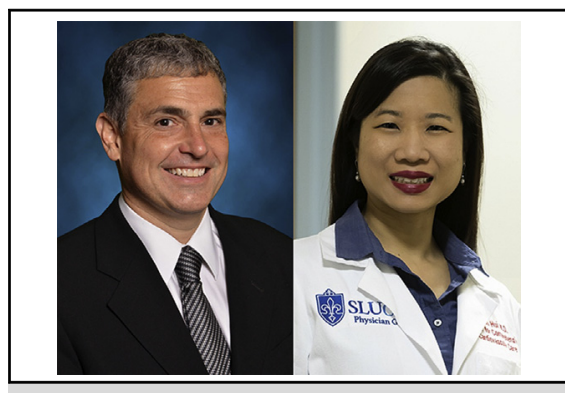

Richard Lee, MD, MBA, and Dawn S. Hui, MD

Central Message

Longitudinal studies of MAR can be challenging to conduct because of variations in practice and follow-up. Randomized trials will provide the best evidence.

See Article page 442

left ventricular dysfunction) and others favoring the LITA group (less chronic obstructive pulmonary disease, prior myocardial infarction, left main or 3-vessel disease). Notably, both the European System for Cardiac Operative Risk Evaluation and in-hospital mortality were significantly worse in patients who received the LITA, suggesting true and clinically significant group differences. Although propensity scores as a covariate were used to adjust for patient factors, group differences in the study end points should be noted. Between LITA and BITA, the follow-up period was differed by approximately 2 -fold (9.7 vs 5.1 years). An important detail to know is that early in the study period the authors preferentially used RA as the second conduit, switching to an "almost obligatory" BITA strategy later in the study. Over time, they also adjusted their skeletonization and sternal wound practices. Any change in clinical practice over time should be considered as a potential confounder in longitudinal studies.

Second, the overall rate of coronary investigation was only $27 \%$ and again differed by group $(68.5 \%$ LITA, $31.5 \%$ BITA). The characteristics of these subgroups may or may not be similar to the total cohort. By using hand calculations of the data in Table 1, it appears that those undergoing coronary investigations differed from the total cohort with regard to gender and multiple risk factors: family history, smoking, diabetes, hemodialysis, and cerebrovascular disease. Observational studies on MAR have shown that among all-comers, significant bias exists. In Benedetto and colleagues' meta-analysis of RITA versus RA studies, ${ }^{5}$ less than $40 \%$ of patients could be propensity score 
matched. Among the 4 methods of using the propensity score, the covariate adjustment method used by Ruttman and colleagues ${ }^{4}$ is not universally accepted as superior and is in fact considered suboptimal by some statisticians. ${ }^{6,7}$ Further, it is difficult to imagine that any statistical method to deal with bias in an observational study can adjust for a major reduction in sample size. In this study, the censure rate was both high and differed by group: $56 \%$ for LITA and $92 \%$ for BITA, leaving only 75 BITA cases for survival analysis at 10 years. Consider that adequately powered RCTs of RA versus SVG require several hundred patients. ${ }^{1-3}$

Although we encouraged continued MAR and commend the authors for their ongoing investigations, the application of various outcomes to variegated subgroups makes it difficult to know how to apply their findings. We have a few other comments regarding their conclusions. First they state that, on the basis of their study findings, MAR using the BITA and RA is the recommended strategy. Yet, BITA + RA was used in only $11 \%$ of the study population and only $6.4 \%$ of those who underwent coronary diagnostic procedures. The analysis showed that the RA is superior to the SVG, but this was primarily by comparing $\mathrm{LITA} \pm \mathrm{RA} \pm \mathrm{SVG}$ with $\mathrm{BITA} \pm \mathrm{SVG}$. If BITA should be the second arterial conduit, this study does not really help us to understand how to use the RA in the context of BITA. Second, the authors discuss the potential for women to more likely benefit from both internal thoracic arteries and RAs; yet the study population was
$87.2 \%$ male overall and more than $90 \%$ male of those with coronary investigations. We should also keep in mind that compared with protocol-based angiography, symptom-based angiography will tend to overestimate graft failure.

The clearest evidence to guide MAR practices will require the results of the randomized Radial Artery Patency and Clinical Outcomes and Arterial Revascularization Trial. Even then, a major question about operator dependence remains because of the currently low rate of MAR practices in general practice.

\section{References}

1. Desai ND, Cohen EA, Naylor D, Fremes SE. A randomized comparison of radialartery and saphenous-vein coronary bypass grafts. N Engl J Med. 2004;351: 2302-9.

2. Collins P, Webb CM, Chong CF, Moat NE. Radial artery versus saphenous vein patency randomized trial. Circulation. 2008;117:2859-64.

3. Hayward PA, Buxton BF. The radial artery patency and clinical outcomes trial: design, intermediate term results and future direction. Heart Lung Circ. 2011; 20:187-92.

4. Ruttman E, Dietl M, Feuchtner GM, Metzler B, Bonaros N, Taggart DP, et al. Long-term clinical outcome and graft patency of radial artery and saphenous vein grafts in multiple arterial revascularization. J Thorac Cardiovasc Surg. 2019;158:442-50.

5. Benedetto U, Gaudino M, Caputo M, Tranbaugh RF, Lau C, Di Franco A, et al. Right internal thoracic artery versus radial artery as the second best arterial conduit: insights from a meta-analysis of propensity-matched data on long-term survival. J Thorac Cardiovasc Surg. 2016;152:1083-91.e15.

6. Deb S, Austin PC, Tu JV, Ko DT, Mazer CD, Kiss A, et al. A review of propensityscore methods and their use in cardiovascular research. Can J Cardiol. 2016;32: 259-65.

7. Garrido MM. Covariate adjustment and propensity score. JAMA. 2016;315: $1521-2$. 